\title{
Wegweisende Veränderungen in der neurologischen (Früh-)Rehabilitation
}

Jürgen Herzog

„Panta rhei - alles fließt“, lautet der bekannte Aphorismus, der seit Jahrtausenden die fortwährenden Veränderungsprozesse unserer Welt in der kürzestmöglichen Formel komprimiert. Die Medizin, von den Grundlagenwissenschaften bis zur klinischen Behandlung, unterliegt diesem Wandel in Form kontinuierlicher Weiterentwicklung in besonderem Maße. Die neurologisch-neurochirurgische Frührehabilitation (NNFR) ist dabei einer der Fachbereiche, der in jüngerer Zeit besonders fundamentalen Veränderungen unterworfen war.

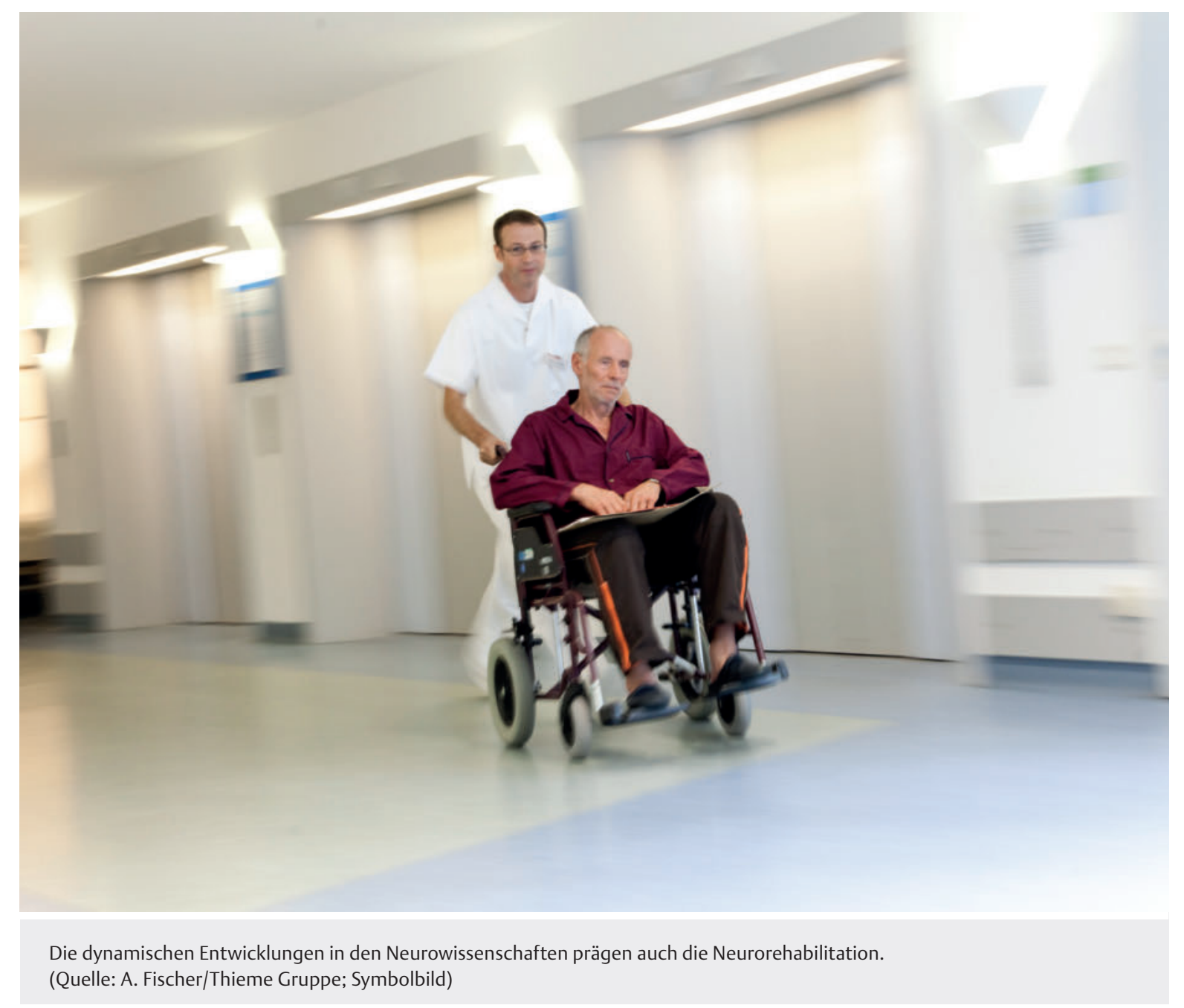


Allein ein Blick auf die Bettenentwicklung verdeutlicht dies: Im Jahr 2000 existierten etwa 1100 Rehabilitationskliniken mit ca. 144000 Betten. Davon waren weniger als 15000 für die Neurologische Rehabilitation, weniger als 1000 Betten für die NNFR und weniger als 100 für beatmete Patienten vorgesehen. 2020 verfügen genauso viele Rehabilitationseinrichtungen über etwa 164000 Betten, jetzt allerdings mit ca. 20000 Betten in der Neurorehabilitation. 5000 Betten sind in der NNFR verortet, davon ca. 1000 Betten mit Patienten zur Beatmungsentwöhnung (Weaning) belegt [1]. Die letzten 20 Jahre haben der NNFR also einen Zuwachs von $500 \%$ an belegten Betten und ca. $1000 \%$ Beatmungsbetten beschert. Ist das rechnerisches Kalkül oder tatsächlicher Bedarf? Und vor allem: Was hat sich darüber hinaus noch alles verändert?

Der folgende Artikel kann nicht alle Aspekte zurückliegender und kommender Veränderungen in der NNFR im Detail beleuchten, sondern muss sich aus Platzgründen auf die wichtigsten beschränken. Die vom Autor vorgenommene Auswahl ist zweifellos subjektiv, zeigt jedoch einerseits die großen Herausforderungen in der gegenwärtigen Zeit, andererseits aber auch Chancen für die Zukunft. Die wichtigsten Aspekte wurden nach Veränderungen der „Medizin“, der „Hygiene“ und der „Gesundheitspolitik und -ökonomie“ kategorisiert.

\section{Veränderungen durch neurologisch fachspezifische Entwicklungen}

\section{Änderungen des Diagnosespektrums und der Fallschwere}

Zur vergleichenden Analyse, wie sich in den zurückliegenden Jahren Rehabilitationsdiagnosen und/oder die Fallschwere verändert haben, eignen sich 2 multizentrische Langzeitverlaufsstudien aus deutschen NNFR-Kliniken: 2002 wurden 1280 Patienten eingeschlossen [2], 2014 insgesamt 784 Patienten [3]. Zu den Top-5-Diagnosen in der NNFR zählen in beiden Erhebungen unverändert der ischämische Schlaganfall (30,2 \% vs. 31,7\% aller Patienten), die intrakranielle Blutung (15,2\% vs. $14,9 \%$ ), das Schädel-Hirn-Trauma (16,1\% vs. 11,5\%) und die hypoxische Hirnschädigung (9,9\% vs. 6,2\%). Am zweithäufigsten ist 2014 jedoch mit 17,1 \% die Critical-Illness-Polyneuropathie (CIP), also ein Krankheitsbild, das 2002 noch nicht vertreten war.

Auch wenn dies in den Studien nicht explizit untersucht wurde, weisen diese meist von Intensivstationen verlegten Patienten eine ausgeprägte Multimorbidität auf, die Ärzten, Pflegepersonal und Therapeuten in der NNFR gleichermaßen die Rehabilitation erschweren. Die Case-MixIndizes (CMI) als Indikatoren der Multimorbidität sind in Frührehabilitationskliniken außergewöhnlich hoch, insbesondere wenn Patienten noch beatmet werden. Unter den Top-10-Kliniken mit der höchsten Fallschwere des Kran-
kenhaus-Reports finden sich seit Jahren ausschließlich spezialisierte Zentren aus der Herzchirurgie und der NNFR.

Hinweise für eine steigende Fallschwere ergeben sich im Vergleich der Studien auch daraus, dass die Patienten vor Verlegung in die NNFR kürzer im primärversorgenden Krankenhaus behandelt wurden (2014 Median 23 Tage, 2002 Median 27 Tage; $p<0,001)$ bzw. dass das Patientenalter (2014: 68,0 \pm 14,8 Jahre, 2002: 60,9 \pm 16,9 Jahre; $\mathrm{p}<0,001)$, der Anteil an beatmeten Patienten in der NNFR (2014: 25,5\%, 2002: 15,2\%; $p=0,007$ ) und die Mortalität (2014: 9,6\%, 2002: 6,4\%; $p=0,031$ ) im Verlauf der Jahre signifikant höher waren. Umso bemerkenswerter ist dahingegen, dass 2014 ein signifikant höherer Anteil an Patienten die weiterführende Rehabilitation (Phase C) erreichte (2014: 38\%, 2002: 33\%; $p=0,024)$.

\section{Demografische Entwicklung}

Die prognostizierte Entwicklung der Altersverteilung hat bereits jetzt Auswirkungen auf die NNFR (s. o., Eintrittsalter in die Frührehabilitation) und wird dies zukünftig in einem noch stärkeren Maße haben. Das Statistische Bundesamt hat ermittelt, dass die Zahl der Menschen ab 67 Jahren bereits zwischen 1990 und 2018 um 54\% von 10,4 Millionen auf 15,9 Millionen stieg. Bis 2039 ist mit einem weiteren Anstieg dieser Zahl auf mindestens 21 Millionen zu rechnen [4]. Ältere bzw. chronisch kranke Menschen sind prädisponiert für neurovaskuläre Erkrankungen, die in der Summe > 50 \% der „typischen“ Diagnosen in der Neurorehabilitation stellen [3].

Aus Registerdaten wird damit gerechnet, dass die Zahl der Schlaganfälle in Deutschland von jetzt knapp 280000 pro Jahr bis 2050 um ca. 68 \% zunehmen wird [5]. Für SchädelHirn-Traumata ist eine analoge Entwicklung zu erwarten: Seit 2013 zeigt sich, dass jedes zweite schwere SchädelHirn-Trauma Folge eines häuslichen Sturzes ist, mit steigender Tendenz. In dieser Gruppe liegt der Altersgipfel jenseits des 60. Lebensjahres. Statistisch gesehen stürzen in dieser Altersgruppe 30 von 100 Menschen mindestens einmal im Jahr schwer, und $12 \%$ erleiden dabei eine Kopfverletzung [6]. Beide Erkrankungen haben als akute Verletzungen des Zentralnervensystems einen hohen Bedarf an spezialisierter Rehabilitation. Es ist also mit einer weiter anwachsenden Zahl von Patienten in der NNFR zu rechnen, deren Altersdurchschnitt steigt.

\section{Demenz als Komorbidität}

Ein weiteres Problem einer überalternden Bevölkerung ist die hohe Inzidenz neurodegenerativer Erkrankungen, deren führendes klinisches Syndrom die Demenz ist. In Deutschland leben nach jüngsten epidemiologischen Schätzungen rund 1,6 Millionen Menschen mit Demenz. Die mittleren Prävalenzraten demenzieller Erkrankungen liegen bei 1,3\% für die Altersgruppe 65-69 Jahre, bei 8,1\% für die 75-79-Jährigen und bei 21,8 \% für die $85-89$-Jährigen [7]. Unter Berücksichtigung des durchschnittlichen 
Patientenalters von 68 Jahren in der NNFR ist es statistisch unumgänglich, dass viele Patienten mit Demenz als (Zweit-)Diagnose auch in neurologischer Rehabilitationsbehandlung sind.

Eine Demenzerkrankung stellt die therapeutischen Konzepte der NNFR vor besondere Herausforderungen: Klassische Strategien des motorischen und kognitiven Lernens funktionieren nicht oder nur eingeschränkt, was die Prognose einer Erholung zu funktioneller Unabhängigkeit im Alltag verschlechtert. Regelmäßige, durchstrukturierte Tagesabläufe, von denen an Demenz Erkrankte grundsätzlich sehr profitieren, lassen sich speziell in Kliniken, aber auch durch interkurrente Erkrankungen (z. B. Infekte) oft nicht realisieren. Die Stimmungs- und Motivationslagen der von Demenz Betroffenen sind labil, ändern sich spontan und korrelieren nur bedingt mit Kriterien des therapeutischen Erfolgs oder Misserfolgs [8]. Die länger anhaltende Entfremdung von vertrauten Umgebungen oder Bezugspersonen während stationärer NNFR führt zu einer erhöhten Rate an Unruhezuständen mit Delir oder Stürzen, mit der Konsequenz des Einsatzes sedierender Medikamente mit z. T. unvorteilhaften Auswirkungen auf die Neuroplastizität (z. B. Neuroleptika).

Generell bleibt festzuhalten, dass Konzepte einer „demenzbegleitenden " Neurorehabilitation derzeit weder mit einer guten Evidenzlage unterlegt noch in Einrichtungen der NNFR flächendeckend verbreitet sind. Gut etablierte Stärken der Rehabilitation, wie z. B. die Einbeziehung von Angehörigen in den Rehabilitationsprozess, könnten hierbei hilfreich sein.

\section{Robotik und Digitalisierung in Therapie und Pflege}

Nur wenige Themen haben die NNFR seit Anfang 2000 in einem ähnlichen Tempo weiterentwickelt wie die Integration robotischer Technologie in die Behandlungsabläufe von Therapie und Pflege. Sogenannte Exoskelette und Endeffektorgeräte zum motorischen Training paretischer Extremitäten gehören in vielen Einrichtungen der neurologischen Rehabilitation zur routinemäßigen Toolbox von Physio- und Ergotherapeuten. „Intelligente“ Software und digital vernetzte Trainingsgeräte ermöglichen therapeutische Spiele („exergaming“) und eine realitätsnähere kognitive Stimulation („enriched environment“) - beides mit zusätzlichen Effekten auf die Patientenmotivation. Schließlich kann „virtuelle Rehabilitation“ mittels telemedizinischer Anwendungen auch in der Neurorehabilitation zum (ferntherapeutisch unterstützten) Eigentraining und damit zur Erhöhung der Therapiefrequenz genutzt werden. Von derartigen Lösungen profitieren nicht nur lokomotorische, sondern auch logopädische oder kognitive Therapieprogramme.

Die therapeutische Wirksamkeit für viele robotische Verfahren ist mittlerweile für funktionale und alltagsrele- vante Parameter (z. B. Arm- und Beinkraft, funktionelle Reichweite, selbstständiges Gehen, Ganggeschwindigkeit, Balance) belegt und in zahlreichen Metaanalysen und systematischen Cochrane Reviews publiziert. Nach Studienlage verbessern sich Paresen durch roboterassistierte Therapie am besten nach ischämischen Schlaganfällen, (traumatischen) Querschnittlähmungen und Multipler Sklerose [9-12].

Es ist bemerkenswert, dass die hohe Wiederholungszahl von robotischen Therapiegeräten die anfänglichen Erwartungen hinsichtlich Effektstärken gegenüber der konventionellen Therapie bislang enttäuscht hat. Auch sind Therapeuten durch Roboter nicht „entbehrlich“ geworden, wie es sich vielleicht mancher Ressourcenoptimierer erhofft hatte. Vielmehr haben die „robotics“ das neurorehabilitative Therapiespektrum sehr sinnvoll ergänzt und gleichzeitig zu einer erheblichen qualitativen Verbesserung in der Durchführung randomisierter kontrollierter Studien (RCT) in den Rehabilitationswissenschaften beigetragen.

Der Vollständigkeit halber sei erwähnt, dass auch in der (therapeutischen) Pflege robotische Systeme klinische Anwendung gefunden haben: Mobilisationsbetten, die die (halb-)automatisierte Vertikalisierung von schwer Betroffenen, z. B. beatmeten Intensivpatienten, in den Stand mit passiven Beinbewegungen kombinieren, sind als Prototypen derzeit in der klinischen Testung und haben sicherlich das größte Potenzial für einen weiter verbreiteten Einsatz. Die medial stärker beachteten „humanoiden Pflegeroboter" sind derzeit wohl eher in der Alten- und häuslichen Pflege vorstellbar als in der Komplexität der NNFR.

\section{Leitlinienentwicklung in der Rehabilitation}

Die Einführung einer Leitlinienkommission in der DGNR seit 2006 mit Etablierung einer ersten fachspezifischen Leitlinie 2009 [13] war ein wichtiger Schritt zur Fokussierung therapeutischer Inhalte der Neurorehabilitation auf evidenzbasierte Verfahren, zur wissenschaftlichen (Neu-) Bewertung der klinischen Praxis und als Impulsgeber zur Standardisierung und Erweiterung des Behandlungsspektrums. Seitdem wuchs die Zahl der publizierten Leitlinien an - aktuell sind auf der Website der Deutschen Gesellschaft für Neurorehabilitation (DGNR) 3 eigene Leitlinien abrufbar; das Portal der Arbeitsgemeinschaft der Wissenschaftlichen Medizinischen Fachgesellschaften e. V. (AWMF) verzeichnet darüber hinaus nicht weniger als 25 weitere Leitlinien anderer Fachgesellschaften, die unter der Mitwirkung der DGNR entstanden sind, weil sie wichtige Themen der Neurorehabilitation berühren (z. B. Spastik, Aphasien, Dysphagien und schwere Bewusstseinsstörungen).

Für die nähere Zukunft bleibt festzuhalten, dass diese Leitlinien zwar vielfach direkte Auswirkungen auf die tägliche Arbeit von Therapeuten in Kliniken und Praxen, bis- 
lang allerdings noch zu wenig Niederschlag in den Ausbildungscurricula therapeutischer Berufe gefunden haben. Hier gilt es konsequent nachzubessern, weil den klassischen, in ihrer Wirksamkeit jedoch nicht belegten „Therapieschulen“ (z. B. nach Bobath oder Vojta) noch Kapazitäten eingeräumt werden, die nach heutiger Kenntnis nicht mehr gerechtfertigt sind.

\section{Veränderungen durch Entwicklungen der Hygiene}

\section{Multiresistente Erreger und Neurorehabilitation}

Bakterielle, multiresistente Erreger (MRE) nehmen in allen Sektoren der Krankenversorgung, insbesondere in stationären Bereichen, seit Jahren kontinuierlich zu. Die NNFR ist von dieser Entwicklung in einem hohem Maße betroffen, weil die Patientenbehandlung durch eine Vielzahl von Risiken charakterisiert ist: vorausgehende, oft komplikationsträchtige Aufenthalte auf Intensivstationen mit hoher nosokomialer Keimlast, rezidivierende Infektionen in der Akut- und Rehaklinik mit jeweils antibiotischer Behandlung, Vorhandensein medizinischer Installationen (z. B. Kathetersysteme), lange Verweildauern, enger körperlicher Kontakt zu einer hohen Zahl verschiedener Kontaktpersonen des therapeutischen Teams, um nur einige der wichtigsten Aspekte zu nennen. Über die quantitative MRE-Prävalenz und nosokomiale Neuinfektionsrate in der NNFR liegen dabei noch wenig bzw. zum Teil widersprüchliche Daten vor, weil zur Häufigkeit von Eingangsund Verlaufsscreenings auf MRE keine verbindlichen Standards existieren.

Rollnik et al. [14] haben über einen 10-Jahres-Zeitraum von 2004-2013 im eigenen Zentrum eine Aufnahmeprävalenz für MRSA (Methicillin-resistenter Staphylococcus aureus) von 11,4\% festgestellt. Die Wahrscheinlichkeit, irgendwann einmal in der NNFR mit einem gramnegativen Erreger, der gegen 3 oder 4 der wichtigsten Antibiotikaklassen resistent ist, (3- oder 4-MRGN) infiziert zu werden, lag dort bei $11,8 \%$. Die tatsächliche MRE-Belastung dürfte jedoch deutlich höher liegen. Systematische Eingangs- und Verlaufsscreenings aus 4 NNFR-Zentren der Schön-KlinikGruppe im Jahr 2015 zeigten, dass bei 36,8\% der Patienten irgendwann während der Rehabilitationsdauer ein MRE nachgewiesen wurde (19\% bei Aufnahme, 12 \% mit Isolationspflicht; unveröffentlichte Daten).

Die Konsequenzen durch Isolationsmaßnahmen sind enorm: Therapien werden erheblich aufwendiger, z. T. nicht möglich (z. B. gerätegestützte oder Therapie in Kleingruppen), die soziale Verarmung der Patienten wächst.

Unter ökonomischen Aspekten führen MRE-Besiedelungen zu einer verlängerten Verweildauer und zu Mehrkosten von
$418 €$ pro Behandlungstag, die bislang nicht in den gängigen Vergütungssystemen abgebildet sind [15].

\section{Rehabilitation bei Covid-19}

Die Pandemie mit SARS-CoV2 seit Anfang 2020 betrifft auch die NNFR in einem außergewöhnlichen Maße. Die Multimorbidität und das hohe Durchschnittsalter machen die Patienten zur besonderen Risikogruppe für eine Infektion bzw. für schwere Verläufe. Die Vielzahl persönlicher Kontakte mit Mitarbeitern des interdisziplinären Teams, die aufgrund der hohen Hilfebedürftigkeit unvermeidliche körperliche Nähe bei pflegerischen und therapeutischen Maßnahmen und nur bedingte Patientencompliance beim Tragen eines Mund-Nasen-Schutzes haben die gewohnten Behandlungsabläufe der NNFR in ein kritisches Licht gerückt (und in nicht wenigen Kliniken völlig zum Erliegen gebracht). Und verschärfte Besucherregeln in Krankenhäusern schließen die Einbeziehung von Angehörigen in den Rehabilitationsverlauf aus.

Andererseits steigt jedoch durch Folgeerkrankungen von Covid-19 der Bedarf an fachspezifischer NNFR zusätzlich: Durch direkte, neurotrope oder indirekte Effekte des SARSVirus werden erhöhte Inzidenzen von Schlaganfällen, Enzephalopathien und Guillain-Barré-Syndromen berichtet [16]. Langzeitbeatmete Patienten nach komplikativen Covid-19-Pneumonien entwickeln Critical-Illness-Polyneuropathien und benötigen rehabilitatives Training.

Im Hinblick auf die steigenden Infektionszahlen bzw. die unklare Dauer des weiteren Pandemieverlaufs sind alle Mitwirkenden der Neurorehabilitation gefordert, sich an der Infektionsprävention, aber auch bei der Bewältigung des fachspezifischen Patientenbedarfs zu beteiligen. Temporäre Schließungen (oder Umwidmung in reine „Abstromkliniken“) von Rehabilitationseinrichtungen, wie noch im Frühjahr 2020 erfolgt, sind gesundheitspolitisch äußerst kritisch zu sehen und sollten vermieden werden.

\section{Veränderungen in der Gesundheits- politik und -ökonomie}

\section{Verweildauer in der stationären Frührehabilitation}

In den beiden schon zitierten deutschen Längsschnittstudien war die Verweildauer in der NNFR 2014 bei den nicht beatmeten Patienten mit einem Median von 37,5 Tagen (49,0 \pm 44,2; N = 562) gegenüber 2002 mit einem Median von 42 Tagen verkürzt (Mittelwert 48,6 \pm 43,9; $N$ = 1077). Auch wenn dieser Trend nicht signifikant war, sind die Ursachen multifaktoriell: Ganz wesentlich hat sicher die Einführung des DRG-Systems im Beobachtungszeitraum dazu beigetragen, das den Druck für eine (kostenoptimierte) Verweildauersteuerung erhöht. Auch die 2014 höhere Mortalitätsrate trägt (z. B. durch unerwartete Todesfälle) zu diesem Effekt bei. Es darf jedoch auch positiv davon aus- 
gegangen werden, dass die wissenschaftsorientierte Professionalisierung der NNFR therapeutische Verfahren effizienter bzw. prognostische Einschätzungen zu einer ressourcenorientierten Patientenselektion sicherer gemacht hat. Dennoch bleibt eine Reduktion dieses medianen Ausmaßes in einem Verlaufszeitraum von nur 12 Jahren bemerkenswert.

Da die Erholung von Schädigungen des Nervensystems auch einer biologischen Verlaufsdynamik unterworfen ist, die nicht beliebig verkürzbar ist, sollte die Verweildauer als Kenngröße der Behandlungsqualität in der NNFR weiterhin aufmerksam beobachtet werden.

\section{Kriterien für den Akutstationären Behandlungsbedarf (ASB)}

In Bayern sind als bislang einzigem Bundesland seit dem 1.9.2012 von einem Expertengremium erstellte und mit den Kostenträgern konsentierte verbindliche Kriterien zur „Rechtfertigung des akutstationären Behandlungsbedarfs in der neurologischen Frührehabilitation“ im Einsatz (sog. „ASB-Checkliste“ [17]). Diese wöchentlich dokumentierten Items sollen operationalisierte Kategorien definieren, ab welchem Zeitpunkt Patienten nicht mehr die Kriterien der NNFR („Phase B“) erfüllen, die durch ihre sozialgesetzliche Verortung als akutstationäre Behandlung (§39SGBV) als Leistung eines Krankenhauses definiert sind.

Eine möglichst präzise Festlegung hierzu war notwendig, weil die Bundesarbeitsgemeinschaft für Rehabilitation (BAR) von 1995 lediglich die Eingangs-, nicht jedoch die Ausschlusskriterien der Phase B festgelegt hatten und es insbesondere in der Abgrenzung zur weiterführenden Rehabilitation („Phase C“, §37 SGB IX) notorische Streitpunkte zwischen den Leistungserbringern und Kostenträgern gegeben hatte. Dem Expertengremium ist es jedoch gelungen, auch Merkmale, die den Rehabilitationsbedarf bzw. das Rehabilitationspotenzial charakterisieren, als behandlungsrelevante Items in die Checkliste zu integrieren. Die ASB-Kriterien wurden seit 2012 zwischenzeitlich zweimal revidiert und sind in der gegenwärtigen Fassung seit 2017 in allen bayerischen NNFR-Einrichtungen im Einsatz. Die Transparenz und die Verbindlichkeit des Katalogs haben zu hoher Akzeptanz bei allen Beteiligten geführt.

Als Schwachpunkt hat sich jedoch eine Subgruppe von Patienten herausgestellt, die nach strenger Auslegung der ASB-Kriterien zu den Verlieren der Vereinbarung zählen: Patienten, die keine akutmedizinischen Kriterien mehr erfüllen, weiterhin Potenzial zu funktionalen Verbesserungen aufweisen, aber durch einen Barthel-Index < 35 Punkte die Eingangsvoraussetzungen in die weiterführende Rehabilitation (noch) nicht erfüllen. Für diese Patienten, deren Anteil in einzelnen NNFR-Kliniken zwischen 0 und $30 \%$ der Gesamtbelegung beträgt, entstand eine Versorgungslücke (sog. „B/C-Lücke“), die entweder zu einer nicht vergüteten Mehrbelastung für die Kliniken oder zu einer vorzeitigen Entlassung rehafähiger Patienten in Pflegeheime führt. Trotz Gesprächen ist eine für alle Seiten befriedigende Lösung dieses Problems noch nicht in Sicht.

\section{Qualitätskriterien für die Neurorehabilitation}

Mit dem Wechsel in das DRG-System (bundesweit seit 2013 abgeschlossen) wurden die Strukturmerkmale für die NNFR flächendeckend durch den OPS-Code 8-552 (Komplexbehandlung „Neurologisch-neurochirurgische Frührehabilitation“) festgelegt. Im Code sind die Anforderungen an Personalqualifikationen, Assessments, Teammitglieder und Therapiemengen zusammengefasst. Die sehr allgemein gehaltenen Basisanforderungen zur Erfüllung (und Abrechnung) des Codes haben bundesweit dazu geführt, dass NNFR nicht nur an etablierten Zentren und Spezialeinrichtungen, sondern in einem wachsenden Anteil auch an Akutkrankenhäusern, in Einzelfällen sogar in nichtneurologischen Facheinrichtungen durchgeführt wird. Es ist davon auszugehen, dass die damit verbundenen Qualitätsunterschiede erheblich sind und letztlich zu Lasten der Patienten gehen, die sich (anders als bei elektiven Behandlungen) ihre Behandlungseinrichtung in aller Regel nicht selbst auswählen können.

Detaillierte und fachorientierte Qualitätskriterien für die NNFR sind deshalb dringend notwendig, bislang jedoch noch nicht konsentiert. Einzig Baden-Württemberg hat seit 2012 eine „Fachplanung Neurologische Frührehabilitation Phase B“ vorgelegt, die das verantwortliche Ministerium bei der Planung und Bewilligung neuer Behandlungseinrichtungen unterstützen soll. Die darin hinterlegten Forderungen orientieren sich jedoch stark an den (veralteten) Kriterien der BAR von 1995 und sind im Hinblick auf Struktur- und Prozessqualität auch nicht präzise genug, um flächendeckend zu einer besseren Behandlungsqualität zu führen. Darüber hinaus existieren bislang nur vereinzelt Positionspapiere von (ärztlichen) Expertengremien (z. B. in Bayern vom „Arbeitskreis Rehabilitation von Schlaganfallpatienten und Schädelhirnverletzten e. V.“), aber keine bundeseinheitliche Regelung. Eine solche wäre jedoch erforderlich, um die überwiegend historisch gewachsenen, regional aber erheblich differenten Unterschiede in der neurorehabilitativen Versorgung auszugleichen und die NNFR flächendeckend und zukunftssicher anzubieten.

\section{Zusammenfassung}

Die dynamischen Entwicklungen in den Neurowissenschaften und der klinischen Neurologie der jüngeren Zeit prägen auch die Neurorehabilitation. Sie führten zu einer Vielzahl von Veränderungen, deren Schwerpunkt insbesondere im Bereich der Frührehabilitation, also der Rehabilitation besonders schwer Betroffener in der Postakutphase lag. Durch eine wachsende Fokussierung auf die Entwöhnung beatmeter Patienten wurden rehabilitative Therapien auf vielen Intensivstationen bzw. intensivmedizinische Behandlungsverfahren in der Frührehabili- 
tation integral miteinander verknüpft. Hierdurch hat sich eine fachliche Spezialisierung der Neurorehabilitation ergeben, die in ihrer Behandlungsdichte und Versorgungsqualität vermutlich weltweit einzigartig sein dürfte. Gerade deshalb sind die Herausforderungen für alle Beteiligten hoch: Bisherige Ausbildungskonzepte für Therapeuten, Ärzte und Pflegekräfte sind überarbeitungsbedürftig, die physische und psychische Arbeitsbelastung aller Mitarbeiter steigt kontinuierlich, und im Prozess der Krankenhausdigitalisierung steht die NNFR noch ganz am Anfang ihrer Entwicklung. Nichtsdestotrotz ist die Hoffnung berechtigt, dass die positiven Veränderungen überwiegen und am Ende die Mehrzahl der Beteiligten von den Veränderungsprozessen profitiert.

\section{Autorinnen/Autoren}

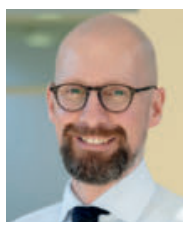

\section{Dr. med. Jürgen Herzog}

Jürgen Herzog ist spezialisiert auf die Bereiche der neurologischen (Früh-)Rehabilitation und der neurologischen Intensivmedizin. Weitere Schwerpunkte stellen die Themengebiete Demenz und „Neuro-Urologie“ dar. Er ist Mitglied in nationalen Fachgesellschaften, sowohl für akutneurologische als auch für rehabilitative Fragestellungen.

\section{Korrespondenzadresse}

\section{Dr. med. Jürgen Herzog}

Chefarzt Neurologische Rehabilitation und Frührehabilitation Arzt für Neurologie

Schön Klinik München Schwabing

Parzivalplatz 4

80804 München

Deutschland

E-Mail: JHerzog@schoen-klinik.de

www.schoen-klinik.de

\section{Literatur}

[1] Mokrusch T, Hrsg. Das Phasenmodell der neurologischen Rehabilitation in Gefahr: Versorgungslücken in der neurologischen Rehabilitation in Deutschland: Phasen C und D - damals und heute. 7. Gemeinsame Jahrestagung der Deutschen Gesellschaft für Neurorehabilitation e. V. und der Deutschen Gesellschaft für Neurotraumatologie und Klinische Neurorehabilitation e. V. Erlangen: 7.12.2018

[2] Pohl M, Berger K, Ketter $G$ et al. Langzeitverlauf von Patienten der neurologischen Rehabilitation Phase B: Ergebnisse der 6-Jahres-Nachuntersuchung einer Multicenterstudie. Nervenarzt 2011; 82: 753-763

[3] Pohl M, Bertram M, Hartwich M et al. Rehabilitationsverlauf von Patienten in der neurologisch-neurochirurgischen Frührehabilitation: Ergebnisse einer multizentrischen Erfassung im Jahr 2014 in Deutschland. Nervenarzt 2016; 87: 634-644
[4] Statistisches Bundesamt, Hrsg. Bevölkerung im Wandel. Annahmen und Ergebnisse der 14. Koordinierten Bevölkerungsvorausberechnung. Wiesbaden: Statistisches Bundesamt; 2019

[5] Foerch C, Misselwitz B, Sitzer M et al. Die Schlaganfallzahlen bis zum Jahr 2050. Dtsch Aerzteblatt 2008; 105: 467-473

[6] Hagebusch P, Pingel A, Kandziora F et al. Das Schädel-HirnTrauma im Erwachsenenalter. Notfallmedizin up2date 2020; 15: $59-74$

[7] Alzheimer Europe. Yearbook 2019. Statistisches Bundesamt. Genesis-Online Datenbank. Fortschreibung des Bevölkerungsstandes: Tab. 12411-0013

[8] Kringle EA, Terhorst L, Butters MA et al. Clinical predictors of engagement in patient rehabilitation among stroke survivors with cognitive deficits: An exploratory study. J Int Neuropsychol Soc 2018; 24: 572-583

[9] Cheung EYY, Ng TKW, Yu KKK et al. Robot-assisted training for people with spinal cord injury: A meta-analysis. Arch Phys Med Rehabil 2017; 98: 2320-2331

[10] Lamers I, Maris A, Severijns D et al. Upper limb rehabilitation in people with multiple sclerosis: A systematic review. Neurorehabil Neural Repair 2016; 30: 773-793

[11] Mehrholz J, Thomas S, Werner C et al. Electromechanicalassisted training for walking after stroke. Cochrane Database Syst Rev 2017; 5(5): CD006185

[12] Mehrholz J, Pohl M, Platz T et al. Electromechanical and robot-assisted arm training for improving activities of daily living, arm function, and arm muscle strength after stroke. Cochrane Database Syst Rev 2018; 9(9):CD006876

[13] Platz T. S3-Leitlinie: Rehabilitative Therapie bei Armparese nach Schlaganfall. AWMF-Leitlinien-Registernummer 080-001; 2020. Im Internet: www.awmf.org/leitlinien/detail/ II/080-001.html; Stand: 10.10.2020

[14] Rollnik JD, Samady AM, Grüter L. Multiresistente Erreger in der neurologisch-neurochirurgischen Frührehabilitation (2004-2013). Rehabilitation 2014; 53: 346-350

[15] Roukens R, Lauster F, Bara M et al. Mehrkosten durch multiresistente Erreger in der Neurorehabilitation. Bundesgesundheitsblatt Gesundheitsforschung Gesundheitsschutz 2017; 60: 1075-1082

[16] Ellul MA, Benjamin L, Singh B et al. Neurological associations of COVID-19. Lancet Neurol 2020; 19: 767-783

[17] Checkliste zur Rechtfertigung des akutstationären Behandlungsbedarfs in der neurologischen Frührehabilitation. Im Internet: www.neuroreha-bayern.de/index.php/die-bayerischen-asb-kriterien; Stand: 10.10.2020

[18] Fachplanung Neurologische Frührehabilitation Phase B. Im Internet: https://sozialministerium.baden-wuerttemberg.de/ fileadmin/redaktion/m-sm/intern/downloads/Downloads_ Krankenhäuser/Neurologische_Fruehrehabilitation_Phase_B_2.0.pdf; Stand: 10.10 .2020

\section{Bibliografie}

DOI https://doi.org/10.1055/a-1267-7534

neuroreha 2020; 12: 153-158

(c) 2020. Thieme. All rights reserved.

Georg Thieme Verlag KG, Rüdigerstraße 14, 70469 Stuttgart, Germany

ISSN 1611-6496 